ACCEPTED ON APJL.

Preprint typeset using $\mathrm{LT}_{\mathrm{E}} \mathrm{X}$ style emulateapj v. 6/22/04

\title{
SEARCHING FOR AGN-DRIVEN SHOCKS IN GALAXY CLUSTERS
}

\author{
A. CAVAliere ${ }^{1}$ AND A. LAPI ${ }^{2,1}$ \\ Draft version October 21, 2018
}

\begin{abstract}
Shocks and blastwaves are expected to be driven driven into the intracluster medium filling galaxy groups and clusters by powerful outbursts of active galactic nuclei or quasars in the member galaxies; the first footprints of shock fronts have been tentatively traced out with X-ray imaging. We show how overpressures in the blasts behind the shock can prove the case and also provide specific marks of the nuclear activity: its strength, its current stage, and the nature of its prevailing output. We propose to detect these marks with the aimed pressure probe constituted by the resolved Sunyaev-Zel'dovich effect. We compute and discuss the outcomes to be expected in nearby and distant sources at different stages of their activity.
\end{abstract}

Subject headings: cosmic microwave background - galaxies: clusters - quasars: general - shock waves

\section{INTRODUCTION}

Density jumps have been recently pinpointed by X-ray imaging of the hot intracluster medium (ICM) that pervades galaxy groups and clusters with average densities around $n \sim 10^{-3} \mathrm{~cm}^{-3}$ and temperatures $T$ in the keV range. These jumps have been interpreted in terms of shock fronts propagating into the ICM out to radial distances $r \approx 0.2 \mathrm{Mpc}$, with Mach numbers around $\mathcal{M} \approx 1.5$, and involving energies up to $\Delta E \approx 3 \times 10^{61} \mathrm{ergs}$ (Mazzotta et al. 2004; Mc Namara et al. 2005; Forman et al. 2005; Nulsen et al. 2005a, b).

Shocks over large scales with such intermediate strengths were specifically expected by Cavaliere, Lapi \& Menci (2002) as marks of the energy being fed back into the ICM by active galactic nuclei (AGNs) when they flare up in member galaxies of a group or cluster. Such events occur when a central supermassive black hole (BH) accretes an additional mass $M_{\bullet} \sim 10^{9} M_{\odot}$; with standard efficiency $\eta \sim 10^{-1}$ for massenergy conversion, this yields over times $\Delta t \sim 10^{8}$ yr energies of order $2 \times 10^{62}\left(M_{\bullet} / 10^{9} M_{\odot}\right)$ ergs.

If these outputs couple at levels $f \sim$ a few percents to the surrounding ICM, they constitute impulsive, considerable additions $\Delta E \approx 10^{61}(f / 5 \%)\left(M_{\bullet} / 10^{9} M_{\odot}\right)$ ergs to its binding energy. In fact, the latter comes to $E \approx G M m / 4 r \approx 3 \times 10^{61}$ ergs in the central $0.2 \mathrm{Mpcs}$ of a cluster, that encompass a dark matter $(\mathrm{DM})$ mass $M \sim 5 \times 10^{13} M_{\odot}$ and an ICM mass fraction $m / M \approx 0.15$. With such appreciable ratios $\Delta E / E$, we expect in the ICM a large-scale blastwave bounded by a leading shock that starts from the host galaxy and moves into the surrounding ICM out to several $10^{-1} \mathrm{Mpc}$.

In fact, the shock Mach numbers are provided in the simple form $\mathcal{M} \approx(1+\Delta E / E)^{1 / 2}$ by the hydrodynamics of the ICM (see Lapi, Cavaliere \& Menci 2005), a good electron-proton plasma which in thermal equilibrium (see $\S 4$ ) constitutes a single "monoatomic" fluid with pressure $P \approx 2 n k T$. For BH masses bounded by $M_{\bullet} \lesssim 5 \times 10^{9} M_{\odot}$ (Ferrarese 2002; Tremaine et al. 2002) relative energy inputs $\Delta E / E \lesssim 1 \mathrm{ob}-$ tain and yield just $\mathcal{M} \approx 1.5$; they also yield standard RankineHugoniot jumps of the post- to the pre-shock density $n_{2} / n_{1}=$ $4 \mathcal{M}^{2} /\left(\mathcal{M}^{2}+3\right) \approx 1.7$, consistent with the X-ray analyses. What other marks will establish such shocks and blasts?

\footnotetext{
${ }^{1}$ Astrofisica, Dip. Fisica Univ. "Tor Vergata", Via Ricerca Scientifica 1, 00133 Roma, Italy.

${ }^{2}$ Astrophysics Sector, SISSA/ISAS, Via Beirut 2-4, 34014 Trieste, Italy.
}

One is constituted by the temperature. This rises sharply across a shock but then falls down in the blast, if nothing else by adiabatic cooling. In any case, resolved spectroscopic measurements of the electron $T$ require many X-ray photons, more than currently available from distant clusters or groups.

Pressure provides another, independent mark; the electron pressure is directly sensed with the SZ effect (Sunyaev \& Zel'dovich 1972). The pressure at the shock is to jump up from the unperturbed level $P_{1}$ by the factor $P_{2} / P_{1}=\left(5 \mathcal{M}^{2}-\right.$ $1) / 4$, which marks a shock from a cold front; moreover, $P$ must retain sufficiently high levels throughout the blast as to propel forward the ICM it sweeps up.

We will see that the radial pressure run $P(r)$ actually rises from the leading shock to an inner "cavity", as long as the blast is driven on by a central AGN. Power must be transmitted from it to the surrounding ICM blast by means of an intervening medium. This may be constituted either by relativistic particles filling up a radiovolume energized by jets (Scheuer 1974; Heinz, Reynolds, \& Begelman 1998); or by another and hotter plasma heated up by the impact of radiation-driven superwinds (see Lamers \& Cassinelli 1999).

Thus SZ pressure probing will have direct implications for the kind and the time-cycle of the AGN outputs, in particular of the radio-loud components.

\section{COMPUTING OVERPRESSURES IN BLASTWAVES}

In predicting pressure distributions, a divide is set by the source time scale $\Delta t$ compared to the blast crossing time $R_{S} / \dot{R}_{s} \approx 2 \times 10^{8} \mathcal{M}^{-1}\left(R_{s} / 0.2 \mathrm{Mpc}\right)$ yr; here $R_{s}$ is the shock radial position, and $\dot{R}_{s}=\mathcal{M} c_{S}$ its velocity in terms of the sound speed $c_{S}=\left(5 P / 3 n m_{p}\right)^{1 / 2}$.

The impact on the ICM of a short lived AGN source may be modeled as an instantaneous central explosion launching the classic self-similar blasts of Sedov (1959) and Parker (1963); these propagate freely at high if decreasing Mach numbers $\mathcal{M}$ (which make $P_{1}$ and gravity irrelevant) into an initial density run $n(r)$. When the latter is provided in the form $n(r) \propto r^{-2}$ by the standard atmosphere in isothermal equilibrium, the pressure $P$ in the blast declines toward the center. Steeper density runs yield an even stronger decline, whilst flatter ones still imply $P<P_{2}$.

But in some sources the observations recalled in $\S 1$ show proximity of the shocks to the edge of the radiovolumes. This indicates blasts being currently driven by the relativistic particles associated with the radiosource, and calls for considering 
AGN outputs sustained during the propagation. A similar indication is provided by the evidence at redshifts $z \gtrsim 0.3$ of superwinds driven by shining quasars (see Stockton et al. 2006). The moderate values observed for $\mathcal{M}$ require including the effects of gravity and finite initial pressure $P_{1}$.

These conditions are aptly modeled with the self-similar blasts derived and discussed by Lapi et al. (2005). A significant testbed is again provided by the isothermal distribution of both the DM and the unperturbed ICM in the gravitational field of the former; their cumulative masses out to $r$ scale like $m(<r) \propto M(<r) \propto r$, and the ICM pressure like $P_{1}(r) \propto n_{1}(r) \propto r^{-2}$.

The overall parameters of the perturbing blast may be derived from the simple "shell approximation", long known to be effective and precise (see Cavaliere \& Messina 1976; Ostriker \& McKee 1988). This treats the blast as a shell containing the mass $m\left(<R_{S}\right)$ swept up out to the radius $r=R_{S}$, and propelled outward by the volume-averaged pressure $\langle P\rangle$ against, we add, the upstream pressure $P_{1}$ and the DM gravity $G M\left(<R_{s}\right) m\left(<R_{s}\right) / R_{s}^{2}$. It leads to the momentum equation

$$
\frac{\mathrm{d}}{\mathrm{d} t}\left[m\left(<R_{s}\right) v_{2}\right]=4 \pi R_{s}^{2}\left[\langle P\rangle-P_{1}\right]-\frac{G M\left(<R_{s}\right)}{R_{s}^{2}} m\left(<R_{s}\right)
$$

in terms of the postshock speed $v_{2}=3\left(\mathcal{M}^{2}-1\right) \dot{R}_{s} / 4 \mathcal{M}^{2}$. Here the pressure terms scale following $P R_{s}^{2}=$ const, as does the gravitational term. Self-similar solutions require also the 1.h.s. to remain constant as the blast moves out; since $m\left(<R_{S}\right) v_{2} \propto R_{S} \dot{R}_{S}-R_{S} c_{S}^{2} / \dot{R}_{S}$ holds (with $c_{S}=$ const), the only consistent solution must satisfy $\dot{R}_{S} \propto c_{S}$. Thus the shock moves outward following $R_{S}=\mathcal{M} c_{S} t$.

On the other hand, the gravitational energy in the DM potential well scales like $G M\left(<R_{S}\right) m\left(<R_{S}\right) / R_{S} \propto R_{s}$, and so do all energies including the binding $E$; this implies for the energy injection $\Delta E(t) \propto t$ to hold, that is, the power $L(t)=$ const is sustained over a crossing time. Then the blast runs unattenuated with $\Delta E(t) / E\left(<R_{s}[t]\right)$ and $\mathcal{M} \approx(1+\Delta E / E)^{1 / 2}$ independent of time and position.

The actual existence of the self-similar solutions is proven from the full hydro equations. By self-similarity these reduce to a set of three ordinary differential equations in the normalized variable $r / R_{s}$, that can be integrated inward of the shock where Rankine-Hugoniot boundary conditions hold, see Lapi et al. (2005). Here we focus on the shape of $P\left(r / R_{S}\right)$ that is plotted in detail in Fig. 1 (left panel).

The point to stress is that $P\left(r / R_{s}\right)$ rises from the shock to the blast inner boundary, namely, the "piston". This is the contact discontinuity separating the ICM from the inner medium in the cavity; the mass $m\left(<R_{p}\right)$ of swept-up gas piles up there, causing the density to diverge mildly whereas the temperature $T(r)$ vanishes. In the blast the two combine to yield a rising $P\left(r / R_{s}\right)$; at the piston this attains a value $P_{p}$ exceeding $P_{2}$ by factors 3-4 (see Fig. 1), larger at lower $\mathcal{M}$.

The rising trend is best understood in the shell approximation on computing the coefficients of Eq. (1), given the scalings above; simple algebra and some labor yield

$$
\frac{\langle P\rangle}{P_{2}}=\frac{4}{5 \mathcal{M}^{2}-1}\left[\frac{5}{4}\left(\mathcal{M}^{2}-1\right)+3\right]=\frac{5 \mathcal{M}^{2}+7}{5 \mathcal{M}^{2}-1} .
$$

The first additive term in the middle expression embodies the contribution to $\langle P\rangle$ from the shell momentum; the second, those from the initial pressure (contributing $1 / 3$ ) plus gravitation (2/3). The final expression shows that $\langle P\rangle / P_{2}$ and hence the monotonic $P(r) / P_{2}$ go to unity for $\mathcal{M} \gg 1$, the result known from Sedov blasts launched into a density gradient with negligible gravity and initial pressure. On the other hand, $\langle P\rangle$ and $P(r) / P_{2}$ increase as $\mathcal{M} \rightarrow 1$; this concurs with the lowering jump $P_{2} / P_{1}$ to yield an overall value $P_{p}$ which decreases slowly with $\mathcal{M}$, as shown by Fig. 1 .

In sum, a driving source excavates in the ICM a cavity with very low inner X-ray emission; this is bounded by the blast (from a rim at the piston to the leading shock) in a cocoonlike topology. Enhanced values $P_{p} / P_{2}$ relate mainly to gravity, important to the ICM as long as all velocities do not exceed $c_{S}$, or the DM velocity dispersion; so at lower $\mathcal{M}$ a relatively stronger push is required to propel the cocoon on.

From the energy standpoint, high values of $P_{p}$ may be viewed as necessary for soaking up - in the form of work $4 \pi R_{s}^{2} P_{p} \dot{R}_{s}$ done at the piston - the power fraction transferred from the source via the inner medium filling the cavity. The fraction is $\alpha \approx 1 / 2$ when the medium is constituted by relativistic particles, see Cavaliere \& Messina (1976), and $\alpha=1$ in the case of a thermalized superwind.

\section{MEASURING OVERPRESSURES WITH THE SZ EFFECT}

The enhanced pressures in the blast can be directly probed with the SZ effect. This arises when photons of the cosmic microwave background (CMB) crossing a cluster are Compton upscattered by the hot ICM electrons; then the black body spectrum of the CMB is tilted slightly toward higher energies.

The resulting intensity change $\Delta I$ of the $\mathrm{CMB}$ radiation at a normalized frequency $x \equiv h \nu / k T_{\mathrm{CMB}}$ is given in the thermal, non-relativistic case (Rephaeli 1995) by

$$
\Delta I=2 \frac{\left(k T_{\mathrm{CMB}}\right)^{3}}{(h c)^{2}} g(x) y .
$$

The effect strength is set by the Comptonization parameter

$$
y(w)=2 \frac{\sigma_{T}}{m_{e} c^{2}} \int_{0}^{\ell_{\max }} d \ell p(r),
$$

just proportional to the electron pressure $p=n k T$ integrated along the line of sight (l.o.s.) at a projected distance $w$ from the cluster center; $\ell_{\max }=\left(R^{2}+w^{2}\right)^{1 / 2}$ applies if the ICM boundary is set at the virial radius $R$.

The spectral shape is encoded in the factor $g(x)$. This has a crossover point at $\nu \approx 220 \mathrm{GHz}$; it is positive for larger $\nu$ with a peak at $\nu \approx 370 \mathrm{GHz}$, and negative for lower $\nu$ with a minimum at $\nu \approx 130 \mathrm{GHz}$. The approximation $g(x) \simeq-2 x^{2}$ applies at the Rayleigh-Jeans end of the CMB spectrum.

As $\Delta I \propto y \propto P$ holds, we expect that in an AGN-driven blast the SZ signal is enhanced from shock to piston, relative to the equilibrium value $\Delta I_{\text {eq }}$ on the l.o.s. grazing the shock. In Fig. 1 (right panels) we show the outcome of our computations based on Eq. (3) and on $P\left(r / R_{S}\right)$ given on the left.

We describe our results beginning with $\nu<220 \mathrm{GHz}$. The rise of $\Delta I / \Delta I_{\mathrm{eq}}$ shown in the top right panel starts up at the shock position $w=R_{s}$ with a high derivative arising from the contribution of the pressure jump $P_{2} / P_{1}$ to the 1.o.s. integral. The subsequent rise toward the piston reflects the further pressure increase into the blast.

Inward of the piston $\Delta I / \Delta I_{\mathrm{eq}}$ rises still further when the inner medium is constituted by a very hot thermal plasma with constant pressure $P_{p}$ (dashed lines); this is closely the case with a thermalized superwind, where $c_{S}$ is high owing to the high pressure $P_{p} \gg P_{1}$ and the low density $n<n_{1}$.

On the contrary, $\Delta I / \Delta I_{\text {eq }}$ declines for $w<R_{p}$ (solid lines) when the cavity within $R_{p}$ is filled with relativistic electrons 

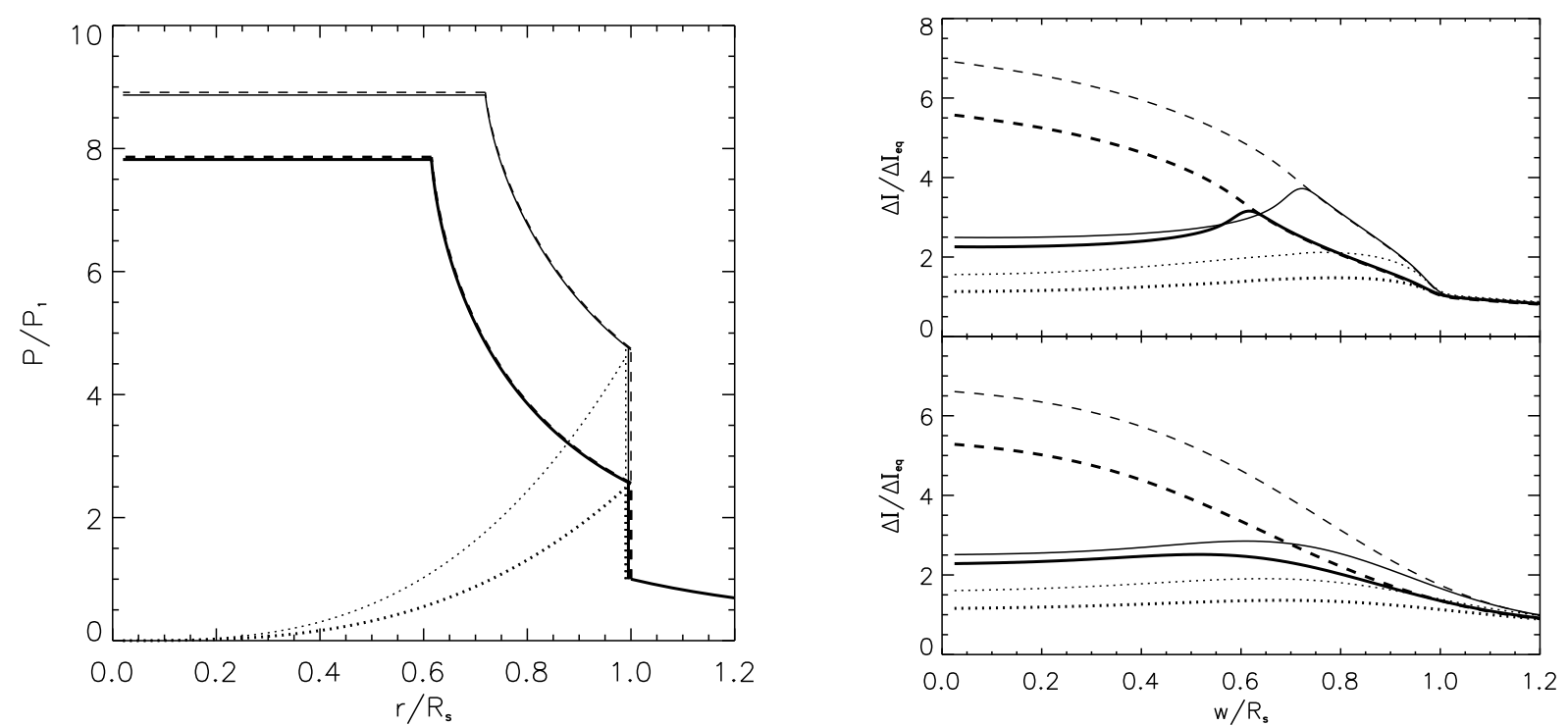

FIG. 1.- Left panel: overpressure in an AGN-driven blastwave; top right panel: enhanced SZ signal (relative to $\Delta I_{\text {eq }}$ on the 1.o.s. grazing the shock) at an instrumental resolution of $1^{\prime \prime}$ in a cluster at $z=0.1 ;$ bottom right panel: same for a resolution of $10^{\prime \prime}$. Thick lines are for Mach number $\mathcal{M}=1.5$, when the piston location is at $R_{p} \approx 0.62 R_{s}$; and thin lines are for $\mathcal{M}=2$, when the piston is at $R_{p} \approx 0.72 R_{s}$. Solid lines are for a blast continuously driven by relativistic particles; dashed lines are for a blast continuously driven by a hot inner plasma; dotted lines are for a standard Sedov-Taylor blastwave launched by a sudden energy release.

(with $c_{S} \approx c / \sqrt{3}$ ). In fact, these provide the pressure $P_{p} \approx 8 P_{1}$ but contribute little to the overall SZ signal on two grounds. First, relativistic pressures $p_{\text {rel }}=P_{p}$ imply low electron densities even with minimal Lorentz factors of a few (see Pfrommer, Enßlin \& Sarazin 2005; Colafrancesco 2005). Second, relativistic electrons are generally inefficient contributors at a given $\nu$ since the tilt they cause in the CMB spectrum is stretched toward high frequencies.

For comparison, we also show the thermal SZ effect produced by free Sedov-Taylor blastwaves launched by a strong but short explosion (dotted lines). Here the pressure declines below $P_{2}$ after the shock jump, so $\Delta I / \Delta I_{\text {eq }}$ rises modestly inward of $w=R_{s}$ and soon decreases.

In fact, the right panels of Fig. 1 show the shapes of $\Delta I / \Delta I_{\mathrm{eq}}$ expected with $\mathrm{SZ}$ instruments having two angular resolutions; in the top panel we show our results smoothed with a Gaussian window of $1^{\prime \prime}$ (about $2 \mathrm{kpc}$ at $z \approx 0.1$ ), and in the bottom panel with $10^{\prime \prime}$ (about $20 \mathrm{kpc}$ ). The latter resolution is currently achieved, while the former will be attained in the near future, see $\S 4$.

For frequencies above $220 \mathrm{GHz}$, similar shapes obtain for $w \leq R_{s}$, except that the non-thermal contribution is generally lower than plotted in Fig. 1. The thermal/non-thermal ratio is maximized at $\nu \approx 370 \mathrm{GHz}$, to decline only at much larger $\nu$. At $\nu \approx 220 \mathrm{GHz}$ only the non-thermal component survives; here the spatial shape of the SZ signal differs significantly on two accounts. First, the rise begins at the piston position $w=$ $R_{p}$ with a sharp transition of $p_{\text {rel }}$ up to $P_{p}$; second, saturation occurs at low levels.

Finally, we discuss why the initial isothermal atmosphere, where $n \propto r^{-\omega}$ applies with $\omega=2$, provides reliable evaluations of the SZ signals from driven blasts. Flatter runs with $\omega<2$ occur in a cluster at $r \lesssim 100 \mathrm{kpc}$; here the gravity is weaker but the mass $m(<r)$ swept up by the blast increases faster, and in the shell approximation Eq. (2) yields for $\langle P\rangle / P_{2}$ a value $6 / 7$ of the isothermal case. Steeper atmospheres corresponding to $2<\omega<2.5$ occur for $r \gtrsim 500 \mathrm{kpc}$; these also yield self-similar blasts propagating at constant $\mathcal{M}$ when acted upon by a fading AGN with integrated energy $\Delta E \propto t^{2(5-2 \omega) / \omega}$. The outcomes include larger pressure jumps but also flatter runs of $P(r)$ at equal values of $\Delta E / E$ (see Lapi et al. 2005); overall, somewhat higher levels of $P_{p}$ obtain.

\section{DISCUSSION AND CONCLUSIONS}

SZ signals are widely measured in clusters at levels $y \approx$ $10^{-4}$, see Reese et al. (2002) and Birkinshaw (2004); Lapi, Cavaliere \& De Zotti (2003) discuss sub-arcmin resolutions to detect the integrated effects of AGN outbursts on the ICM of clusters and groups. Here we have focused on SZ signals resolved at levels of $10^{\prime \prime}$ or better, to probe the structure and the dynamics of the shocks and blasts so produced.

The SZ probe is best used in scanning the ICM around density jumps selected in X-rays. In fact, the bremsstrahlung surface brightness proportional to $n^{2}$ is well suited for pinpointing density jumps and providing positions and Mach numbers of candidate shocks. But measuring $T$ from X-ray spectroscopy must contend with paucity of photons and the narrow post-shock range where $T$ exceeds the unperturbed value.

So in conditions of low surface brightness (outskirts or distant structures) the SZ effect will lend a strong hand by unveiling the other key observable, namely, the overpressures behind the shocks; this is due to three circumstances. First, pressures are sensed directly through the parameter $y \propto P$. Second, $y$ is independent of $z$ for sources wider than the instrumental beamwidth. Third, we expect (see Fig. 1) thermal pressures to rise throughout a blast continuously driven over a crossing time; from shock to piston at radii $40-30 \%$ smaller, $P(r)$ rises up to values $P_{p} \approx 8-9 P_{1}$, considerably larger than the shock jump $P_{2} / P_{1} \approx 2.6-4.8$ for $\mathcal{M} \approx 1.5-2$; this is due to the dynamical stress in running blasts.

This rising behavior of $P(r)$ is just opposite to the run down from shock to center expected for free blasts launched by short-lived AGN activity. We compare in Fig. 1 the results we expect; they constitute an aim particularly interesting for 
shocks of intermediate $\mathcal{M}$ pinpointed in close proximity to a radiovolume or around a currently shining AGN. Clear study cases will be provided by clusters or groups in quiet conditions, with no sign of outer merger induced dynamics.

What is needed for SZ probing at $z \approx 0.1$ is a resolution around $10^{\prime \prime}$ in the upper $\mu$ wave band, already approached with the Nobeyama radiotelescope (Kitayama et al. 2004, also http: //www.nro.nao.ac.jp/index-e.html/). Upcoming instruments such as CARMA (http://www.mmarray.org/) will do better; at resolutions of a few arcsecs, blasts in clusters at $z \approx 0.5$ may be probed at levels comparable to the top right panel of Fig. 1. ALMA (http://www.alma.nrao.edu/) with its planned resolutions around $1^{\prime \prime}$ and sensitivities down to $1 \mu \mathrm{K}$, will do better yet both in the $\mu$ wave and in the submm band. Following the noise analysis by Pfrommer et al. (2005), a $5 \sigma$ detection with ALMA of the thermal SZ signal we focus on will require scanning a limited area in the vicinity of an X-ray preselected position for a few hours per cluster.

We add that once kpc scales will be resolved, two-fluid effects will be interesting as may arise from disequilibrium between the electrons and ions (see Ettori \& Fabian 1998; Fabian et al. 2006). They may cause a gradual rise of the electron pressure in front of the shock, to converge behind it with the declining ion pressure toward the equilibrium values considered above.

Here we stress three issues. First, how common are shocks in clusters? The frequent occurrence of quenched cooling flows argues for widespread shocks driven over some Gyrs by central AGNs injecting energies $\Delta E$ in excess of the cooling losses (see Bîrzan et al. 2004; Nulsen et al. 2005a, b).
Second, in what prevailing mode does an AGN release the driving energy $\Delta E$, in mechanical form and radio jets or in radiation-driven superwinds? To a first approximation the mode little affects the total energy injected, based on the simple rule $\Delta E \sim \alpha f \eta c^{2} \dot{M}_{\bullet} \Delta t \sim$ const that we extract from Churazov et al. (2005); that is, mechanical energy and jets gain on grounds of coupling efficiency $(\alpha f \approx 1 / 2$ vs. a few $\%$ ) what they lose to radiation on grounds of $\eta \dot{M}_{\bullet}$. Conversely, the shock energetics alone will not distinguish the mode.

Third, what other probe may help? The specific SZ probing we propose can trace the distinguishing features of the injection: its timescale (past or ongoing), and its prevailing content (relativistic particles or photons). The SZ effect resolved at levels of $10^{\prime \prime}$ or better will directly detect the mark of a blast launched or driven by a powerful AGN, namely, the hydro overpressure jumping up at the shock and sustained throughout the blast. But when the radiosource drive persists over the transit time the pressure actually rises in the blast, and the SZ signal is enhanced; it is boosted up by plasma in the cavity if the drive is helped by AGN superwinds.

These outcomes are independent of model details, rather they depend on a few overall parameters: the relative injection energy $\Delta E / E$, evaluated from $\mathcal{M}$; the active time of the AGN compared with the blast crossing time; the injection mode, whether dominantly in mechanical or radiative energy. We conclude that SZ measurements concurring with the $\mathrm{X}$-ray imaging can effectively probe the injection mode, the dynamics of the blasts, and the history of the driving AGN sources.

We thank our referee for helpful comments.

\section{REFERENCES}

Bîrzan, L., Rafferty, D.A., McNamara, B.R., Wise, M.W., \& Nulsen, P.E.J., 2004, ApJ, 607, 800

Birkinshaw, M., 2004, Carnegie Observatories Astrophysics Series Vol. 3 (Cambridge: Cambridge Univ. Press)

Cavaliere, A., Lapi, A., \& Menci, N., 2002, ApJ, 581, L1

Cavaliere, A., \& Messina, A. 1976, ApJ, 209, 424

Churazov, E., Sazonov, S., Sunyaev, R., Forman, W., Jones, C., \& Böhringer, H., 2005, MNRAS, 363, L91

Colafrancesco, S., 2005, A\&A, 435, L9

Ettori, S., \& Fabian, A.C., 1998, MNRAS, 293, L33

Fabian A.C., et al., 2006, MNRAS, 366, 417

Ferrarese, L., 2002, ApJ, 578, 90

Forman, W., et al. 2005, ApJ, 635, 894

Heinz, S., Reynolds, C.S., \& Begelman, M.C., 1998, ApJ, 501, 126

Kitayama, T., et al., 2004, PASJ, 56, 17

Lamers, H.J.G.L.M., \& Cassinelli, J.P., 1999, Introduction to Stellar Winds (Cambridge: Cambridge University Press), Ch. 12

Lapi, A., Cavaliere A., \& Menci, N., 2005, ApJ, 619, 60

Lapi, A., Cavaliere, A., \& De Zotti, G. 2003, ApJ, 597, L93
Mazzotta, P., Brunetti, G., Giacintucci, S., Venturi, T., \& Bardelli, S., 2004, Jour. of the Korean Astron. Soc., 37, 381

McNamara, B.R., Nulsen, P.E.J., Wise, M.W., Rafferty, D. A., Carilli, C., Sarazin, C.L., \& Blanton, E.L., 2005, Nature, 433, 45

Nulsen, P.E.J., Hambrick, D.C., McNamara, B.R., Rafferty, D., Bîrzan, L., Wise, M.W., \& David, L.P., 2005a, ApJ, 625, L9

Nulsen, P.E.J., McNamara, B.R., Wise, M.W., \& David, L.P., 2005b, ApJ, 628,629

Ostriker, J.P., \& McKee, C. 1988, Rev. Mod. Phys., 60, 1

Parker, E.N. 1963, Interplanetary Dynamical Processes (New York: Wiley)

Pfrommer, C., Enßlin, T.A., \& Sarazin, C.L., 2005, A\&A, 430, 799

Rephaeli, Y., 1995, ARAA, 33, 541

Reese, E.D., et al., 2002, ApJ, 581, 53

Scheuer, P.A.G., 1974, MNRAS, 166, 513

Sedov, L.I., 1959, Similarity and Dimensional Methods in Mechanics (New York: Academic Press)

Stockton, A., Fu, H., Henry, J.P., \& Canalizo, G., 2006, ApJ, 638, 635

Sunyaev, R.A., \& Zel'dovich, Ya.B., 1972, Comm. Astroph. Sp. Sc., 4, 173

Tremaine, S., et al. 2002, ApJ, 574, 740 